\title{
LCA studies comparing beverage cartons and alternative packaging: can overall conclusions be drawn?
}

\author{
Eva von Falkenstein • Frank Wellenreuther • \\ Andreas Detzel
}

Received: 22 June 2010 / Accepted: 1 July 2010 /Published online: 27 July 2010

(C) Springer-Verlag 2010

\begin{abstract}
Background and purpose Numerous life cycle assessments (LCAs) have been conducted on the environmental impacts of beverage packaging systems. With such a potentially rich source of knowledge available, it seemed worthwhile to conduct a comprehensive evaluation of those existing studies. This paper describes a recent 'meta analysis', whose goal it was to provide a structured overview of LCAs on beverage cartons and other packaging systems from past years in order to answer two key questions: (1) Is it possible to draw general conclusions regarding the environmental performance (in terms of strengths and weaknesses) of beverage cartons in comparison to alternative packaging systems from these existing LCAs? (2) If certain trends arise across these LCA studies regarding the environmental performance of beverage cartons compared to other packaging systems for beverages, what can be said on their validity and limitations?

Methods The meta analysis presented covers 22 LCA studies, all of which fulfil three criteria: (1) full life cycle approach, (2) beverage carton must be among the products evaluated in study, and (3) comparative approach. Each of these studies was categorised either as a core study (if focussed on Europe, conducted in 2000 or later, and peer reviewed) or as a basic study. Next to providing detailed comparisons of the analysed studies, the full report on the meta analysis was designed to allow a quick understanding of their main characteristics (or 'profiles'). Similarities and differences were highlighted both in terms of results and the
\end{abstract}

E. von Falkenstein $(\varangle) \cdot$ F. Wellenreuther $\cdot$ A. Detzel

IFEU-Institut für Energie- und Umweltforschung

Heidelberg $\mathrm{GmbH}$,

Wilckensstr. 3

69120 Heidelberg, Germany

e-mail: eva.falkenstein@ifeu.de applied methodologies (e.g., key settings) and the validity and limitations of the findings were stated. Additionally, further environment-related topics of special interest to stakeholders in the beverage packaging industry were addressed.

Results, discussion, and conclusions For certain environmental impact indicators/inventory categories, the LCA studies covered in this meta analysis indicate general trends regarding the performance of beverage cartons versus alternative packaging systems. For climate change, cumulated energy demand/fossil resource consumption, and acidification, all regarded by the majority of all studies, beverage cartons mostly have the most favourable results, while in terms of land use for forestry, they clearly require the largest area. For summer smog and terrestrial eutrophication, the result 'pattern' points towards a favourable picture for beverage cartons; however, fewer LCA studies provide results for these impact categories. For other environmental aspects, where the results of the analysed studies vary strongly, no clear pattern can be made out. Several aspects were covered in too few LCA studies in order for an overall trend or lack thereof to become visible, and still others - which in part have been receiving increased attention in the past years - are not addressed in any of the analysed core or basic studies.

Keywords Beverage cartons · Beverage packaging · Environmental impacts · Inventory categories .

Methodology $\cdot$ Review of LCA studies

\section{Background, aim, and key questions}

In the past years, numerous life cycle assessments (LCAs) on the environmental impacts of beverage packaging 
systems have been conducted. The Alliance for Beverage Cartons \& the Environment and the World Wide Fund for Nature asked Institut für Energie- und Umweltforschung Heidelberg GmbH (IFEU, Institute for Energy and Environmental Research Heidelberg, Germany) to conduct a comprehensive evaluation of those existing studies, with a special focus on beverage cartons. The aim of this metaanalysis was to provide a structured overview of LCAs on beverage cartons (BCs) and other packaging systems which have emerged in the past 10 years. The exercise may be repeated with further LCAs in the future.

The key questions this meta-analysis aims to answer are:

1. Is it possible to draw general conclusions regarding the environmental performance (in terms of strengths and weaknesses) of beverage cartons in comparison to alternative packaging systems from these existing LCAs?

2. If certain trends arise across these LCA studies regarding the environmental performance of beverage cartons compared to other packaging systems for beverages, what can be said on their validity and limitations?

The full report, which is available upon request from the commissioner $^{1}$, presents a comprehensive technical evaluation and overview of the findings and conclusions of the individual LCA studies included in the meta-analysis. It allows a quick understanding of the analysed studies' main characteristics, highlighting both similarities and differences not only in terms of results but also regarding the applied methodologies. In the following, we aim to provide a condensed account of the approach taken and the outcomes of the work.

\section{Material: LCA studies covered by the meta-analysis}

\subsection{Selection and categorisation}

The meta-analysis covers 22 LCA studies, identified by means of a thorough search of relevant sources and accessible to the members of the project panel. All of these studies fulfil the following criteria:

- full life cycle approach (i.e. no cradle-to-gate studies) as basic principle of evaluation,

- a beverage carton must be among the products evaluated,

${ }^{1}$ For further information and contact details, please see the Alliance for Beverage Cartons \& the Environment (ACE) website under http:// www.ace.be/index.jsp?nodeId $=10073 \&$ isoCode $=$ de.
- comparative approach (comparison of different products, i.e. a beverage carton and at least one other packaging type).

Based on both formal and content-related criteria, each of these studies was categorised either as a core study or as a basic study. The seven core studies, focussing on Europe, peer-reviewed and conducted in 2000 (the publication year of the ISO standard series on LCA (ISO 2006a, b), which was revised in 2006) or later were analysed in depth (key characteristics, results and key settings). Fifteen further studies, which do not fulfil these stricter criteria but were likely to be relevant to the meta-analysis, were analysed on a more general level (key characteristics and results).

Note that in contrast to this article, the full report (see footnote) includes a list of the individual studies that were analysed. Some of the LCAs analysed in the meta-analysis are confidential and not (yet) publicly available. However, the information disclosed in the report was approved by those persons or organisations that provided the respective documents to IFEU for the purpose of the meta-analysis and for referencing in the full report.

\subsection{Key characteristics}

The studies that were analysed were produced within a range of 12 years and cover various geographic scopes, as Table 1 shows. Although in this meta analysis the majority of publications are of European origin, several other countries (markets) are represented, such as USA, Canada and Indonesia.

The analysed studies also show great variation in terms of fill goods (beverage products) and volumes (packaging sizes) as well as types of packaging that were examined. The majority of reports that clearly state which beverages the respective LCA covered, focus on juice products and/or milk. However, a few studies also address packaging for other drinks such as water and wine. In terms of packaging

Table 1 Years of LCA studies/reports covered in the meta-analysis as well as respective numbers and geographic scopes

\begin{tabular}{lll}
\hline Year & Number of LCAs & Geographic scopes \\
\hline 1997 & 1 & EU \\
1999 & 2 & Germany \\
2000 & 1 & Germany \\
2002 & 2 & Germany, Indonesia \\
2003 & 3 & Italy, Sweden \\
2004 & 2 & Germany, Switzerland \\
2005 & 2 & Italy, Netherlands \\
2006 & 3 & Germany, USA/Canada \\
2007 & 2 & Switzerland, USA \\
2008 & 4 & Finland, France, Spain, Turkey \\
\hline
\end{tabular}


size, both single- and multi-serving sales units are covered; most studies specifically examine at least one 1-litre container. Table 2 provides a summarised overview of the fill goods, volumes and packaging types covered in the analysed LCA studies.

The environmental impact indicators regarded by the authors of the analysed LCAs include both resource- and emission-related aspects and in addition to these, several inventory level categories were also addressed. Table 3 gives an overview of these impact indicators and inventory categories. Where terminologies vary between reports, we applied a single 'name' for the respective group.

\subsection{Results}

In accordance with the key questions of this work, we summarised the results documented in the analysed LCA studies per fill good for all comparisons that concern beverage cartons. A detailed overview of these findings from the individual studies is included in the full meta-analysis report, which is available upon request from the commissioner. In the following sections of this article, we will focus on presenting our approach for the comparison and interpretation of this large number of results as well as the conclusions.

\section{Method: comparison and interpretation of results}

3.1 Focus on relative performances (of one specific product among several)

For the most part, the specific figures (quantitative results) from different studies could not be compared directly as each of the 22 analysed LCA studies had been conducted within specific framework conditions and there were significant variations regarding the key settings of each study. We therefore chose a format for presenting the results, which expresses the relative performance of the beverage carton (BC) in the comparisons made by the analysed LCA studies, i.e. its ranking compared to the regarded packaging alternatives. This relative performance of the packaging product at the centre of attention was expressed by a ratio (e.g. 1/3). The first value (numerator, here ' 1 ') indicates the ranking of the $\mathrm{BC}$ among all packaging systems compared in the respective case group within the original LCA study, with the product ranking first having the lowest environmental impact in this comparison. The second value (denominator, here ' 3 ') stands for the number of different packaging types (and variants) compared in the respective case group. As an example, the ratio $1 / 3$ thus means that the $\mathrm{BC}$ shows the lowest environmental impact of three examined packaging systems.

Furthermore, a colour code was implemented to facilitate the quick classification of a specific result as one of three general cases:

(a) the $\mathrm{BC}$ shows (one of) the lowest environmental impact(s) of all packaging types compared in the respective case group,

(b) the $\mathrm{BC}$ shows neither the lowest nor the highest environmental impact, i.e. it lies between or is very close to those of other packaging types and

(c) the $\mathrm{BC}$ shows (one of) the highest environmental impact(s) of all packaging types compared in the respective case group.

As two of the selected LCA studies are actually two complementary parts of one large study, and the second part

Table 2 Fill goods (beverages), volumes (packaging sizes) and package types covered in the analysed LCA studies

\begin{tabular}{llll}
\hline Fill goods & Number of LCAs & Fill volumes and packaging types & $>500 \mathrm{~mL}$ \\
\cline { 3 - 4 } & & $\leq 500 \mathrm{~mL}$ & Beverage carton \\
Juice products & 11 & Beverage carton & Bottles: PET, glass \\
& 11 & Bottles: PET, glass & Beverage carton \\
Milk (ambient, fresh/chilled) & 3 & Beverage carton & Bottles: PET, HDPE, glass \\
Water & 2 & Bottles: PET, HDPE, glass & Beverage carton \\
& & Beverage can & Bottles: PET, glass \\
Wine & & Bottles: PET, glass & Beverage carton \\
& & Beverage carton & Bottles: PET, glass \\
Unspecified/other & Bottles: PET, glass & Beverage carton \\
& & Beverage carton & Bottles: PET, HDPE, PVC, glass \\
\end{tabular}

PET polyethylene terephthalate, HDPE high density polyethylene, $P V C$ polyvinyl chloride 
Table 3 Environmental impact indicators and inventory level categories covered in the analysed LCA studies (numbers indicate how many reports address each aspect)
Environmental impact indicators

\begin{tabular}{lll}
\hline Resource related & Emission related & \\
\hline Energy resource consumption (13) & Climate change (22) & Cumulated energy demand (14) \\
Land use (8) & Acidification (18) & Waste (5) \\
Water consumption (3) & Eutrophication (18) & Traffic (3) \\
Abiotic resource consumption (2) & Summer smog (14) & \\
& Ecotoxicity (5) & \\
& Human toxicity (5) & \\
& Ozone depletion (4) & \\
& Winter smog (2) & \\
& Odour (2) & \\
\hline
\end{tabular}

Note that the terminology does not always correspond with that of the original LCA report environmental impact in 3 case groups (e.g. different fill volumes) examined by two studies. An example of how this number code was used can be seen in Table 4.

In the full report, the same colour code was used as for the detailed result documentation (see previous section).

\subsection{Focus on robust results and comparisons}

In order to achieve robust comparative conclusions from this meta-analysis, some results (from the analysed LCA studies) were excluded from interpretation:

- results, for which the original report does not clearly specify the fill good,

- impact indicators and inventory level categories that are analysed by less than three LCA studies for a specific fill good: abiotic resource consumption, winter smog, odour and Traffic,

- fill good categories examined by less than three LCA studies: wine, ice tea and water (the latter is covered in three of the 22 analysed reports, however two of these are actually consecutive parts of one large study, and the second part does not directly compare different packaging systems).

With this, the final conclusions apply to packaging systems for the fill good categories juice, ambient and fresh (chilled) milk. Due to the omissions described above and in Section 3.1, they are ultimately based on 16 of the previously selected 22 LCA studies.

\section{Results: conclusions and discussion}

\subsection{Conclusions drawn from the meta-analysis}

In the following, the conclusions derived from the examined LCAs are presented concerning the environmen-

tal performance (in terms of strengths and weaknesses) of for at least one case group. For example, the indication 3 thus means that the $\mathrm{BC}$ is found to have the lowest 
Table 4 Numbers of case groups (1st value) and LCA studies (2nd value=subscript) ranking the beverage carton in similar ways within the respective comparisons to alternative packaging types; beverage category 'Juice' includes nectar and ice tea

\begin{tabular}{|c|c|c|c|c|}
\hline \multicolumn{2}{|c|}{ Relative environmental impact of beverage carton in regarded product comparison: } & \multirow{2}{*}{$\frac{\text { Lowest }}{17_{10}}$} & \multirow{2}{*}{$\begin{array}{l}\text { Neither lowest nor highest } \\
1_{1}\end{array}$} & \multirow{2}{*}{$\begin{array}{l}\text { Highes } \\
-\end{array}$} \\
\hline Climate change & Juice $(J)$ & & & \\
\hline & Ambient milk (AM) & $8_{8}$ & - & - \\
\hline & Fresh milk (FM) & $9_{7}$ & - & $1_{1}$ \\
\hline \multirow[t]{3}{*}{ Acidification } & $\mathrm{J}$ & $14_{9}$ & $2_{2}$ & $1_{1}$ \\
\hline & $\mathrm{AM}$ & $6_{6}$ & - & - \\
\hline & FM & $10_{8}$ & - & - \\
\hline \multirow[t]{3}{*}{ Cumulated energy/fossil resource consumption } & $\mathrm{J}$ & $16_{8}$ & $1_{1}$ & - \\
\hline & $\mathrm{AM}$ & $6_{6}$ & - & - \\
\hline & FM & $7_{6}$ & - & $1_{1}$ \\
\hline \multirow[t]{3}{*}{ Land use (forestry) } & $\mathrm{J}$ & - & - & $12_{6}$ \\
\hline & $\mathrm{AM}$ & - & - & $3_{3}$ \\
\hline & FM & - & - & $4_{3}$ \\
\hline \multirow[t]{3}{*}{ Summer smog } & $\mathrm{J}$ & $7_{4}$ & $1_{1}$ & $4_{2}$ \\
\hline & $\mathrm{AM}$ & $4_{4}$ & - & - \\
\hline & FM & $6_{5}$ & - & - \\
\hline \multirow[t]{3}{*}{ Terrestrial eutrophication } & $\mathrm{J}$ & $10_{6}$ & $1_{1}$ & - \\
\hline & $\mathrm{AM}$ & $2_{2}$ & $1_{1}$ & - \\
\hline & FM & $7_{6}$ & - & - \\
\hline
\end{tabular}

Exemplification: ' 32 ' listed in the rightmost column means that the beverage carton was found to have the highest environmental impact in three case groups (e.g. with different fill volumes) that were examined by two studies

beverage cartons (BCs) in comparison to alternative packaging systems. As explained in the previous Section 3.3, they cover those results we deemed to be of appropriate quality, and thus apply only for three beverage categories: juice (including nectar and ice tea), ambient and fresh (chilled) milk. Furthermore, not all environmental aspects covered by the analysed LCA studies are addressed.

Where possible, a short discussion of the identified patterns (e.g. trends in the ranking of the $\mathrm{BC}$ ) and exceptions is included and at the end of the section, Table 4 exemplifies how these patterns were identified. Indications concerning the validity and limitations are given here only where they apply to a specific environmental indicator/ category. For further information on the findings' validity, see section 4.3.

\subsubsection{Climate change}

All analysed LCA studies assess the greenhouse gas emissions and in the great majority of cases, the $\mathrm{BC}$ is attributed with the lowest impact (42 case groups from 20 studies). One study comes to the opposite result when comparing the $\mathrm{BC}$ exclusively to a multi-use glass bottle for fresh milk; in all other studies, the BC is compared to oneway packs.

The trend described above applies in spite of a variety of settings covered in the individual LCA studies and is generally reflective of both non-carbonated soft drinks (incl. juice) and milk.

\subsubsection{Acidification}

Acidifying impacts are examined by most LCA studies. For the beverage category milk, the $\mathrm{BC}$ is always attributed with the lowest impact. For juice, the results show a trend in the same direction - with one exception where a polyethylene terephthalate (PET) bottle is attributed with a smaller impact than the analysed BC. This may have to do with the fact that here, a higher recycling rate is assumed for PET bottles than for the BC, and that a 100:0 allocation factor is used for credits in open-loop recycling (i.e. the upstream system is given $100 \%$ of the credits).

This favourable picture for beverage cartons (15 LCA studies find BCs to have the lowest acidifying impacts of all packaging types compared in 33 case groups) applies for a variety of different settings covered in the individual LCA studies, similar to the findings regarding Climate change.

\subsubsection{Cumulated energy (total, non-renewable, fossil)/fossil resource consumption}

For the beverage categories juice and milk, the consumption of non-renewable/fossil resources, often examined with a focus on energy carriers, is assessed by all except three of 
the regarded LCA studies. In all cases but one, the $\mathrm{BC}$ is attributed with the lowest impact. The analysis of a case group for fresh milk where the $\mathrm{BC}$ is attributed with the highest resource consumption is the exception. In this case, a multiuse glass bottle is the alternative packaging type, while in the other studies the $\mathrm{BC}$ is compared to one-way packs.

\subsubsection{Water consumption}

The use of water is only explicitly covered in the results sections of three studies (exclusively for the fill good milk), and the meta-analysis shows a mixed picture regarding the relative performance of BCs. With this, no sound conclusions can be drawn on the ranking of the $\mathrm{BC}$ concerning this environmental issue.

\subsubsection{Land use (forest)}

Only some of the analysed LCA studies explicitly regard the occupation of land surface by forestry systems (juice: half of the respective studies, milk: about a fourth). In those studies that do so, the BC is always associated with a larger use of forest area than the compared alternative packaging systems ( 25 case groups within seven studies). This is a direct consequence of its material composition: around three quarters of the $\mathrm{BC}$ consist of paperboard from wood fibre. Alternative products, however, use relatively little biomass feedstock or none at all.

One other study also attributes the $\mathrm{BC}$ with the highest 'land use' value while not specifying the type of land use (i.e. it is unclear, which share of this land is forest area).

\subsubsection{Land use (sealed, landfill)}

Land uses other than forestry (such as sealed area and landfills) are examined by very few of the LCA studies examined in the meta-analysis, and the results show a mixed picture. For juice containers, only three studies explicitly cover this aspect, among them one that ranks the $\mathrm{BC}$ as the least favourable product regarding the 'use of nature'. In this case, the comparison is made with a multiuse glass bottle with a high reuse rate (37 cycles).

One other study also attributes the $\mathrm{BC}$ with the highest 'land use' value while not specifying the type of land use (see above, forest area). Due to the small number of studies addressing the topic, no sound conclusions can be drawn on the ranking of the $\mathrm{BC}$ regarding this environmental issue.

\subsubsection{Summer smog}

Over half of the analysed LCA studies examine summer smog. The respective results indicate a possible trend towards attributing the $\mathrm{BC}$ with the lowest impact. For the fill good milk, the results show no exceptions. For juice, four out of six studies produce results, which support this picture but at the same time, three cases do not rank the $\mathrm{BC}$ first. In one study, a PET bottle is attributed with a more favourable performance than the examined BC. This may have to do with the fact that here, a higher recycling rate is assumed for PET than for the BC, and that a 100:0 allocation factor is used for open-loop recycling (i.e. the upstream system is given $100 \%$ of the credits). Furthermore, the analysed PET bottle is comparably light-weight. Concerning the two LCA studies attributing the $\mathrm{BC}$ with the highest impact, a derivation of explanations is not possible from the parameters analysed in the meta-analysis.

\subsubsection{Eutrophication}

Eutrophication potentials of packaging systems are examined by over half of the LCA studies covering juice and milk. Overall, the results could indicate a possible trend towards attributing the $\mathrm{BC}$ with the lowest impact. However, only half of the analysed LCA studies that address eutrophication distinguish between different subcategories of this environmental issue. Those studies that specify terrestrial and/or aquatic eutrophication show different pictures:

- Terrestrial eutrophication is explicitly covered by only four of the analysed LCA studies for juice, all of which attribute the $\mathrm{BC}$ with the lowest impact (in seven case groups). For milk, only two studies specify results for terrestrial eutrophication, which is insufficient to draw any sound conclusions.

- Aquatic eutrophication is only explicitly examined by five studies for the beverage category juice, and by four for milk: the results show a very mixed picture. For juice, three studies attribute the $\mathrm{BC}$ with the greatest relative impact, while another study comes to the opposite result and yet another, which regards two case groups, comes to a different result for each. Of the four studies that explicitly analyse the aquatic eutrophication of milk packaging, two rank the $\mathrm{BC}$ as the most favourable and two as the least favourable packaging type. Therefore, no evidence of any trend can be derived from the meta-analysis.

\subsubsection{Ecotoxicity}

Only three of the LCA studies covering juice and milk explicitly examine ecological toxicity, in all cases attributing the $\mathrm{BC}$ with the lowest impact. This applies for different settings, competitive packaging systems and geographic scopes. However, due to the small number of studies addressing the topic, it cannot be verified within the meta-analysis whether the favourable relative ranking of the $\mathrm{BC}$ for this environmental issue reflects a general trend. 


\subsubsection{Human toxicity}

Human toxicity is only examined by three of the LCA studies covering packages for juice and milk, and the results show a mixed picture. In total three different indicators are applied (PM10, heavy metals, carcinogenic risk) across the studies, and the results vary strongly depending on the indicator used and the case group. Therefore, no evidence of any trend can be derived from the meta-analysis.

\subsubsection{Waste}

Only very few LCA studies examine the amounts of final waste generated by the examined packaging systems (four for the fill good category milk and only one for juice), showing a mixed picture regarding the relative performance of the BC. Three of these studies attribute at least one of the examined $\mathrm{BC}$ variants with the greatest relative impact, two with the lowest and in one, the result for the $\mathrm{BC}$ is similar to that of the only analysed packaging alternative. Therefore, no evidence of any trend concerning waste can be derived from the meta-analysis.

\subsection{Conclusions on trends (numerical presentation of selected results)}

For several environmental impact/inventory level categories, the results from the analysed LCA studies reveal patterns, which indicate (possible) trends regarding the relative performance of beverage cartons compared to alternative packaging systems. Climate change, cumulated energy demand/fossil resource consumption, and acidification are each addressed by the majority of the analysed studies. The respective results for juice and milk indicate a clear trend of beverage cartons having the most favourable results. In contrast, beverage cartons clearly require the largest area of land use for forestry, an inventory level category highlighted by much fewer studies. For summer smog (addressed by about half of the studies) and for terrestrial eutrophication (covered by only a few of the analysed LCAs) result 'patterns' are less obvious, however they do point toward favourable pictures for beverage cartons. For other environmental aspects, where the results of the analysed studies vary strongly, no trends can be made out. Several aspects were covered in too few LCA studies in order for an overall trend or lack thereof to become visible, and still others - which in part have been receiving increased attention in the past years - are not addressed in any of the analysed core or basic studies.

Table 4 exemplifies how the numerous results from the analysed LCA studies were structured in order to reveal the patterns mentioned above, the key question being: 'How many case groups (comparisons) in how many studies come to similar rankings?'

\subsection{Remark on validity and limitations}

The conclusions presented in the previous Section 4.1 are valid within the described framework conditions and the introduction of this article. They are subject to limitations (a) arising from the methodological approach taken within this meta-analysis and (b) inherent in the original LCA studies that were analysed - and thus partly beyond the influence of the authors of the meta-analysis. Examples of both kinds are listed and described in detail in the full report, which is available upon request from the commissioner.

\subsection{Further environmental issues (outlook} and recommendations)

Several other environment-related topics have been of growing interest to various stakeholders in the public and industryinternal debate on LCAs. Due to the fact that wood fibre is the main raw material used in beverage cartons, the following three are of special importance for the beverage carton and liquid packaging board (LPB) industry, i.e. the commissioners of the meta-analysis. Within the supply/process chain of LPB, the origin of wood sources and biodiversity issues relate to forestry while process water use is usually discussed in the context of pulping, bleaching and paperboard manufacturing, or, in short, the board mill operations.

In addition to the two key questions, which the metaanalysis primarily sought to answer, we therefore also surveyed the material with the aim of finding out to which extent those aspects were taken into account in the examined LCA studies. All three topics are subject to current developments of LCA impact assessment methodology, however to date no agreed methods and suitable inventory data are available. Consequently, only few LCAs have included them so far-applying different methodologies. In the following, each of these topics is covered in brief: its potential relevance in LCAs is addressed and its inclusion in the analysed LCA studies is highlighted.

\subsubsection{Origin of wood resources}

Inventory data on the consumption of wood resources is necessary to ensure a consistent life cycle inventory. Without additional information regarding the origin of the wood sources (including the applied forest management practices) used in a product system, it is impossible to evaluate the overall environmental or 'sustainability' impact of wood sourcing in a specific supply chain.

None of the LCA studies included in the meta-analysis covers this topic within the environmental impact assessment applied or the interpretations derived. Yet, in the future, this issue could become crucial for the credibility of LCA results for product systems based on biomass resources. Some of the 
involved parties already have implemented a certification by the Forest Stewardship Council of their chains of custody for paperboard going into (European) beverage cartons. We highly recommend including such frameworks and exploring other sources of adequate information to improve the data availability for future LCAs.

On a general note, today's LCAs typically also lack information on the origin of other non-wood resources like petroleum and metals. However, information on the origin of a resource is a key to assessing its sustainable use(s).

\subsubsection{Biodiversity}

So far, loss of biodiversity is rarely applied as an environmental category as such in LCA impact assessments. On the other hand, it is considered a relevant indicator for assessing the environmental quality of land use. Primarily, land use is accounted for at the inventory level, measured as the land area occupied for different activities along the supply chain of a product. Taking the land use and its qualities into account is particularly important if the examined product system relies strongly on the use of biomass.

Various attempts have been made to make the type of land use operational for LCAs based on the respective environmental qualities, for example by 'measuring' the loss of biodiversity. Again, this requires more detailed information on the specific types of land use involved in the supply chain of a product system: the actual number of species, for example, along with respective reference values. It is obvious that for this kind of approach, a minimum of spatial data is necessary at the inventory level. The currently existing LCA databases do not yet provide such a level of detail.

A different approach for assessing the loss of biodiversity is built on existing certification systems. It is based on the assumption that biomass from certified sources implicitly guarantees the conservation of biodiversity within the agricultural and forestry systems it comes from. The precondition is however, that the respective certification scheme is suited for measuring and evaluating the biodiversity of a specific area. Similarly to the approach described in the previous paragraph, this one also needs further methodological development and is closely linked to the availability of more spatial information at the inventory level.

None of the LCA studies included in the meta-analysis explicitly addresses loss of biodiversity as a separate impact category. Given the increasing demand for renewable raw materials from 'sustainable production', all entities in the supply chains concerned, among them the beverage carton industry, are advised to investigate options of implementing assessment methods particularly for forestry and agricultural systems within practical LCA work.

\subsubsection{Water use}

Currently, the input of water into product systems is again receiving special attention in the context of LCAs. To date, water input most typically is dealt with in the life cycle inventory without further characterisation. However, information on water input in publicly available inventory datasets is not consistent. Some datasets just report on 'water' in general, others differentiate sources (surface water, ground water, rain water, etc.) or areas of water application (process water, cooling water, etc). This inconsistency makes it difficult to even sum up the water input at different points in the life cycle to a total water input of the entire product system.

To date, water use has not been given much attention in life cycle assessments. In order to be able to address water input at impact category level, information is required on the scarcity of water at individual points of water demand along the supply chain of a product. Here, approaches have been proposed to "measure" water scarcity in countries or geographic regions by relating the respective gross water consumption to the availability of renewable water resources.

Furthermore, besides the points mentioned above, a clear common terminology is needed and currently evolving at international level.

Five of the analysed LCA studies address water input into packaging systems:

- two reports document 'use of water' at the level of inventory results,

- two other reports document 'water consumption', in part at the level of inventory results and

- one study documents 'water extraction'.

For future LCA work, we recommend investigating methodological options of how water input can be dealt with at the impact assessment level. This question is particularly relevant when a product system involves water-intensive raw materials.

\section{References}

ISO (2006a) Environmental management - Life cycle assessment Principles and framework (ISO 14040:2006). International Organization for Standardization, Geneva

ISO (2006b) Environmental management - Life cycle assessment Requirements and guidelines (ISO 14040:2006). International Organization for Standardization, Geneva 